\title{
Effectiveness of Subcutaneous Growth Hormone in HIV-1 Patients with Moderate to Severe Facial Lipoatrophy
}

\author{
Miwako Honda ${ }^{1}$, Akira Yogi ${ }^{2}$, Naoki Ishizuka ${ }^{3}$, Ikumi Genka ${ }^{1}$, Hiroyuki Gatanaga ${ }^{1}$, \\ Katsuji Teruya ${ }^{1}$, Natsuo Tachikawa ${ }^{1}$, Yoshimi Kikuchi ${ }^{1}$ and Shinichi Oka ${ }^{1}$
}

\begin{abstract}
Objective: To evaluate effect of recombinant human growth hormone (rhGH) among HIV-infected adults with moderate to severe facial lipoatrophy as a side effect of long-term antiretroviral treatment.

Design: A prospective open-label study

Methods: Twenty-five HIV-1 patients with moderate to severe facial lipoatrophy who had been on antiretroviral treatment for more than 18 months were enrolled. rhGH $(5 \mathrm{mg})$ was given every other day for 6 months. After treatment was completed, the participants were followed up for 6 months. Facial lipoatrophy was evaluated by computed tomography at months $0,3,6$ and 12 .

Results: Nearly all participants (24 of 25 ) completed the study. The sum of bilateral soft tissue thickness at the level of zygomatics at months $0,3,6,12$ were $7.23,8.59,8.35,8.60 \mathrm{~mm}$, respectively. There was significant improvement from baseline in month $3(\mathrm{p}=0.009)$ and month $12(\mathrm{p}=0.021)$. In the 6 months of followup, the soft tissue showed no significant decrease. Several side effects including diarrhea, arthralgia, myalgia, mastalgia and hand numbness were seen, which were self-limited and transient.

Conclusion: $\mathrm{rhGH}$ is effective and relatively safe for moderate to severe facial lipoatrophy. Its effect was sustained at least for 6 months after the cessation of rhGH.
\end{abstract}

Key words: HIV, antiretroviral treatment, lipoatrophy, recombinant human growth hormone

(DOI: 10.2169/internalmedicine.46.6122)

\section{Introduction}

The prognosis of HIV-1 patients has been remarkably improved by highly active antiretroviral therapies (HAART). However, many patients have suffered from long-term adverse effects including lipoatrophy, which markedly interferes with their quality of life $(1,2)$. Recombinant human growth hormone (rhGH) has been used for patients with fat redistribution syndrome and has shown favorable outcomes in trunk, limbs or lipid profile (3-5). Among this syndrome, a significant percentage of patients has experienced facial lipoatrophy, which has one of the strongest impacts on their quality of life $(6,7)$. This prospective study was undertaken to focus on the effect of subcutaneous growth hormone in HIV-1 patients with moderate to severe facial lipoatrophy.

\section{Method Patients}

The study was designed as a pilot, non-randomized prospective open-label study for HAART induced lipoatrophy. Patients who have been treated with HAART for more than 18 months with moderate to severe facial lipoatrophy were recruited from the clinic at the AIDS Clinical Center in International Medical Center of Japan, located in the center of Tokyo. All participants were screened from July 1, 2003 through Dec 31, 2003. The grade of lipoatrophy was defined based on the criteria of the facial lipoatrophy severity scale (8) (Table 1). Based on the scale, grade I is defined as mild, grades II and III as moderate and grade IV as severe facial lipoatrophy.

Other inclusion criteria are; 1) aged 20 to 65,2 ) on stable antiretroviral regimen at least 6 months prior to enrollment,

${ }^{1}$ AIDS Clinical Center, International Medical Center of Japan, Tokyo, ${ }^{2}$ Radiology, International Medical Center of Japan, Tokyo and ${ }^{3}$ Community Health and Medicine, International Medical Center of Japan, Tokyo

Received for publication July 11, 2006; Accepted for publication November 21, 2006

Correspondence to Dr. Miwako Honda, mihonda@imcj.acc.go.jp 
Grade I: mild and focalized facial lipoatrophy

Grade II: deeper and longer atrophy, with facial muscles beginning show through

Grade III: atrophic lesion even deeper and wider, with the muscles clearly showing

Grade IV: lipoatrophy covers wide area, extending toward the eye sockets, and the facial skin lies directly on the muscles

Table 2. Characteristics of Patients at Entry

\begin{tabular}{ll}
\hline Patients $(\mathrm{n})$ & 25 \\
$\quad$ Male & 21 \\
$\quad$ Female & 4 \\
Age (years) & $38.6(24-55)$ \\
Duration of antiretroviral treatment (years; mean, SD) & $4.79(1.61)$ \\
Mean CD4 (cells $/ \mathrm{mm}^{3}$ ) (mean, SD) & $468.4(202.2)$ \\
Number of patients with viral load less than 50 copies $/ \mathrm{ml}$ & $22(88 \%)$ \\
\hline
\end{tabular}

3) have not been treated with rhGH or other anabolic steroid in the past 6 months, 4) fasting blood glucose $<126 \mathrm{mg} / \mathrm{dl}$ $(9), 5)$ no obvious current opportunistic infection.

The number of participants was set according to the conditions of the pilot study, quality control of safety and monitoring, and the amount of rhGH provided by the manufacturer; 25 patients, 21 males and 4 females, aged between 24 and 54 years were enrolled the study. The average duration of antiretroviral treatment was 4.79 years, and 22 patients were with undetectable (less than 50 copies $/ \mathrm{ml}$ ) viral load, the average CD4 was 468.4 cells $/ \mathrm{mm}^{3}$ (468.4+/- 202.2) (Table 2).

\section{Procedures and Statistical Analysis}

rhGH $(5 \mathrm{mg})$ was given subcutaneously every other day for 6 months. After the completion of the rhGH injection, patients were followed up for 6 months. The observation period was a total of 12 months. Antiretroviral treatment was continued throughout the study. rhGH was provided by Serono Japan Co., Ltd.

The primary endpoint was the change in the soft tissue thickness of the face. All patients had computed tomography (CT) of the face at the level of maxillary sinus, zygomatic arch and mandibular ramus at months $0,3,6$ and 12 (10). Preliminary, interobserver variability was evaluated. CT of the face was performed and the soft tissue thickness in each slice was measured by two independent radiologists. Kappa value was 0.754 , which was considered acceptable agreement. Upon the result, the facial soft tissue in all slices was measured by one radiologist. Analysis of variance was used as the statistical method. Multiple comparison of DunnettHsu analysis was used to test the difference between each soft tissue thickness in CT in comparison with their base- line.

The secondary endpoint includes body composition assessed by body mass index, circumflex of limbs and percentage of body fat, blood test with lipid profile, glucose and liver function test, CD4 and viral load which were measured at each visit at months 0, 3, 6 and 12. Patients were also asked to complete questionnaires on their quality of life. Facial photographs were taken at each visit.

The Ethics Committee of the International Medical Center of Japan approved the study. All participants were informed about the study and gave written consent prior to the participation.

\section{Result}

Of the 25 participants, one patient had severe diarrhea within 1 month and withdrew from the study. 24 completed the study, however, the digital CT data of 4 patients was partially lost due to technical error. Therefore, the CT scans of 20 participants were analyzed.

The sum of bilateral facial soft tissue at the level of zygomatics at months $0,3,6,12$ were $7.23 \mathrm{~mm} ; 8.59 \mathrm{~mm}, 8.35$ $\mathrm{mm}$ and $8.60 \mathrm{~mm}$, respectively (Fig. 1). Dunnett-Hsu analysis of adjusted multiple comparison of least squares means found significant improvement of soft tissue thickness from the baseline in the month $3(\mathrm{p}=0.009)$ and month $12(\mathrm{p}=$ 0.021). Even after the completion of rhGH injection at month 6 , the soft tissue at the level of zygomatics showed no significant decrease for the follow-up period.

There was no significant change in the circumference of arm and thigh, and liver function, CD4 nor HIV viral load during the study. BMI and lipid profile also showed no change except for glucose between months 0 and 6 , both of which were within the normal limit (Table 3). 
Table 3. Change of Laboratory Characteristics

\begin{tabular}{lllllll} 
& month 0 & month 3 & month 6 & month 12 & $p$ (month 0 to 6) & $p$ (month 0 to 12) \\
\hline BMI (mean, (SD)) & $20.8(2.6)$ & $21.3(2.3)$ & $21.1(2.9)$ & $21.5(2.8)$ & 0.188 & 0.009 \\
Glucose (mean, (SD)) & $89.8(15.5)$ & $96.5(17.7)$ & $101.5(17.9)$ & $92.6(16.3)$ & $<0.0005$ & 0.592 \\
Triglyceride (mean, (SD)) & $288.5(146.5)$ & $299.0(157.9)$ & $247.3(158.5)$ & $319.3(254.6)$ & 0.211 & 0.593 \\
Total Cholesterol (mean, (SD)) & $201(45.2)$ & $199.9(48.0)$ & $191.2(41.5)$ & $195.5(16.3)$ & 0.114 & 0.431 \\
\hline
\end{tabular}

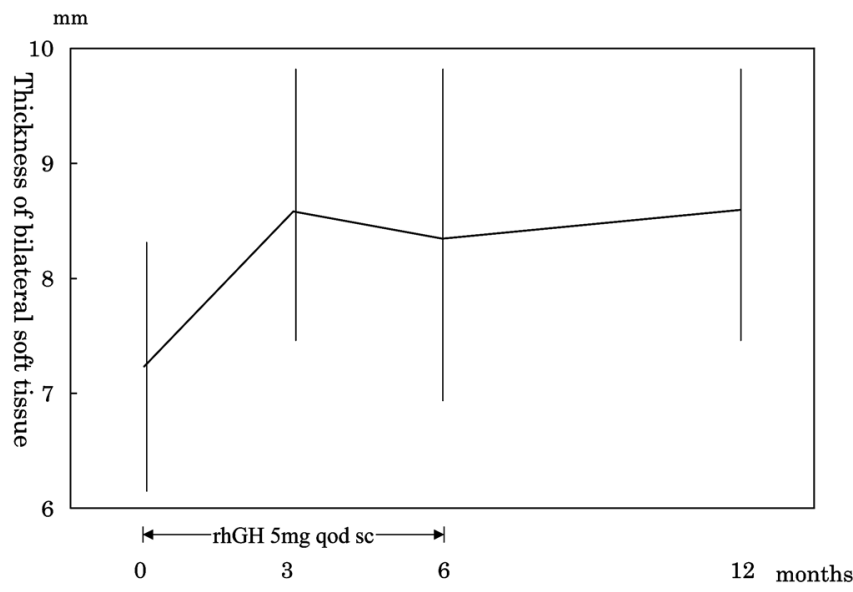

Figure 1. Thickness of bilateral soft tissue at the level of zygomatics, $95 \%$ CIs.

The quality of life questionnaire revealed that 19 of 25 patients had felt some improvement in their appearance and they were satisfied with the results.

Several adverse events were noted during the study. One patient withdrew due to severe diarrhea. His symptoms gradually subsided after the cessation of rhGH. Ten patients experienced transient self-limited mild arthralgia or muscle ache, 3 male patients had mild mastalgia or enlargement of breast tissue, and one had right hand numbness. All symptoms have resolved after the completion of the $\mathrm{rhGH}$.

\section{Discussion}

Facial lipoatrophy is one of the long-term adverse effects of HAART for which standard treatment is not yet found and it severely interferes with the patient's quality of life. Several studies using surgical intervention have been reported to show limited transient effects (8). Some studies have reported the effect of growth hormone for lipoatrophy or lipodystrophy in relation to the total body composition or glucose metabolism $(3-6,11,12)$. However, there has been no report of rhGH effect focusing on facial lipoatrophy. This prospective study was designed as a single arm pilot study to focus on the change of facial soft tissue thickness and the maintenance effect with the use and after the cessation of rhGH. All of the participants were followed and evaluated by a single institute, which had the benefit of close clinical monitoring for patient safety and quality assurance of the study. The soft tissue at the zygomatics showed significant improvement of lipoatrophy in month 3 with rhGH and in the observation period without rhGH in month 12 . The effect was sustained for 6 months after the cessation of rhGH. This CT- based evaluation method is accurate and reproducible. In particular, the Kappa value of 0.754 showed that the interobserver variability is minimal.

Considering the fact that many patients who have been on long-term antiretroviral treatment in the era of HAART suffer from lipoatrophy $(6,7)$, this study proved that rhGH has a significant and sustained effect on the improvement of facial lipoatrophy.

Other clinical parameters, including BMI, liver function test, lipid profile, serum glucose, viral load and CD4 showed no significant change. Although severe diarrhea had led a patient to withdraw from the study, other side effects (arthralgia, myalgia, mastalgia and hand numbness) were self-limited and transient. Upon consideration of these results, rhGH can be considered relatively safe to use.

While the standard use of rhGH is $5 \mathrm{mg}$ subcutouneously every day, several studies have shown that low-dose rhGH was effective in visceral adipose tissue and preventive of the change in glucose tolearanve or insulin sensitivity $(11,12)$. Our study protocol reduced the frequency to every other day with the standard dose, aiming to prevent changing glucose tolerance and to reduce the cost while expecting the maximal effect. The result showed that there was no significant change in glucose intolerance and lipid profile on rhGH. The outcome is quite encouraging.

A potential weakness of the study is the cost of rhGH. For this trial, rhGH was provided by the manufacturer. However, the total cost of rhGH of this study was about 37,000 USD for 6 months use for one patient. The national health insurance of Japan approves of rhGH only for the treatment of HIV-related wasting syndrome. None of the participants met the criteria at the entry of this study. The cost effectiveness of the use of rhH for facial lipoatrophy will require further discussion.

\section{Conclusion}

rhGH is effective and relatively safe for moderate to severe facial lipoatrophy while it is in use and after the cessation. Patients were satisfied with the outcomes of subcutaneous injection of rhGH. The cost effectiveness of rhGH for facial lipoatrophy needs further discussion. 


\section{References}

1. Carr A, Samaras K, Burton S, et al. A syndrome of peripheral lipodystrophy, hyperlipidemia and insulin resistance in patients receiving HIV protease inhibitors. AIDS 12: F51-F58, 1998.

2. Koutkia P, Canavan B, Breu J, Torriani M, Kissko J, Grinspoon S. Growth hormone releasing hormone in HIV-infected men with lipodystrophy: a randomized controlled trial. JAMA 292: 210-218, 2004.

3. Wanke C, Gerrior J, Kantaros J, Coakley E, Albrecht M. Recombinant human growth hormone improves the fat redistributionsyndrome (lipodystrophy) in patients with HIV. AIDS 13: 2099-2103, 1999.

4. Schwarz JM, Mulligan K, Lee J, et al. Effects of recombinant human growth hormone hepatic lipid and carbohydrate metabolism in HIV-infected patients with fat accumulation. J Clin Endocrinol Metab 87: 942-945, 2002.

5. Kotler DP, Muurahainen N, Grunfeld C, et al. Effects of growth hormone on abnormal visceral adipose tissue accumulation and dyslipidemia in HIV-infected patients. J Acquir Immune Defic Syndr 35: 239-252, 2004(erratum in: J Acquir Immune Defic Syndr 35: 381, 2006).

6. Moyle GJ, Daar ES, Gertner JM, et al. Growth hormone improves lean body mass, physical performance, and quality of life in sub- jects with HIV-associated weight loss or wasting on highly active antiretroviral therapy. J Acquir Immune Defic Syndr 35: 367-375, 2004.

7. Nicholas PK, Kirksey KM, Corless IB, Kemppainen J. Lipodystrophy and quality of life in HIV: symptom management issues. Appl Nurs Res 18: 55-58, 2005.

8. James J, Carruthers A, Carruthers J. HIV-associated facial lipoatrophy. Dermatol Surg 28: 979-986, 2002.

9. American Diabetes Association. Diagnosis and classification of diabetes mellitus. Diabetes Care 29: S43-S48, 2006.

10. Padilla S, Gallego JA, Masia M, Gutierrez F. Single-slice computed tomography and anthriopometric skinfold analysis for evaluation of facial lipoatrophy in HIV-infected patients. Clin Infect Dis 39: 1848-1851, 2004.

11. Lo JC, Mulligan K, Noor MA, et al. The effects of low-dose growth hormone in HIV-infected men with fat accumulation: a pilot study. Clinical Infectious Diseases 39: 732-735, 2004.

12. Andersen O, Haugaard SB, Flyvbjerg A, et al. Low-dose growth hormone and human immunodeficiency virus-associated lipodystrophy syndrome: a pilot study. Eur J Clin Invest 34: 561-568, 2004.

(C) 2007 The Japanese Society of Internal Medicine http://www.naika.or.jp/imindex.html 